\title{
Enhanced photovoltaic performance in nanoimprinted pentacene-PbS nanocrystal hybrid device
}

\author{
D. M. N. M. Dissanayake, A. A. D. T. Adikaari, ${ }^{a)}$ and S. R. P. Silva \\ Advanced Technology Institute, University of Surrey, Guildford, Surrey, GU2 7XH, United Kingdom
}

(Received 2 January 2008; accepted 13 February 2008; published online 6 March 2008)

\begin{abstract}
Pentacene and $\mathrm{PbS}$ nanocrystal bilayer photovoltaic devices are fabricated after the pentacene layer is subjected to nanoimprinting using a laser textured silicon stamp. Increased short circuit current densities are observed for the imprinted devices, which are attributed to increased charge mobility in the pentacene film caused by the decrease in the intermolecular distances during nanoimprinting. This work is consistent with previous reports where hydrostatic pressure induced mobility increases have been observed in polyacenes under gigapascal pressure regimes. It is believed that the pentacene film undergoes localized high pressures during nanoimprinting, giving rise to the increased hole mobilities. (C) 2008 American Institute of Physics. [DOI: 10.1063/1.2890848]
\end{abstract}

Semiconducting organic materials are suitable for low cost, large area, and flexible optoelectronic device fabrication. Recent reports demonstrate up to $6 \%$ optical to electrical power conversion efficient organic photovoltaic devices designed using the bulk heterojunction architecture. ${ }^{1}$ However, to increase the power conversion efficiency $(\eta)$ beyond the envisaged market entry figure of $10 \%$, further enhancement of the photovoltaic device properties, such as short circuit current $\left(J_{\mathrm{sc}}\right)$, open circuit voltage $\left(V_{\mathrm{oc}}\right)$, and fill factor, is essential. Incorporation of semiconducting nanocrystals, which demonstrate size dependent band gaps into organic photovoltaics, can potentially increase the light absorption from a wider part of the terrestrial solar spectrum. Such hybrid photovoltaic devices demonstrating light harvesting up to $1600 \mathrm{~nm}$ region have so far been reported in the literature. ${ }^{2}$ These devices, however, operate at lower $\eta$ mainly due to poor charge transport properties of the nanocrystals and unfavorable energy level offsets between the donor-acceptor active layers. In an attempt to rectify these shortcomings, promising device architectures consisting of small molecule organic dyes (pentacene) and infrared absorbing $\mathrm{PbS}$ nanocrystals (ns-PbS) have been investigated. ${ }^{3,4}$ Alternatively, power conversion efficiency of organic photovoltaic devices can be increased by enhancing exciton dissociation at the heterojunction through nanostructuring. Recent reports indicate up to twofold increase in the $J_{\mathrm{sc}}$ of pentacene $/ \mathrm{C}_{60}$ bilayer devices nanostructured by a technique involving nanoimprinted lithography (NIL). ${ }^{5}$ We report incorporation of the NIL technique to increase the device performance of the pentacene/nc-PbS hybrid device architecture. We observe that $J_{\text {sc }}$ increases remarkably by two orders of magnitude after nanoimprinting, which is attributed to an increased in the charge mobility within the hole transporting layer. It is proposed that alternative phenomena, such as increased exciton dissociation, are not facilitated by the imprinting conditions used for this work.

Twice sublimed pentacene $(99.99 \%)$ was purchased from H.W. Sands and used without further purification. nc-PbS were synthesized with a narrow size distribution according to a method reported by Hines and Scholes. ${ }^{6}$ Prior to device fabrication, the oleic acid ligands of nc-PbS were exchanged

${ }^{\text {a)} E l e c t r o n i c ~ m a i l: ~ d . a d i k a a r i @ s u r r e y . a c . u k . ~}$ to shorter butylamine ligands. To perform ligand exchange, the dots were dried under nitrogen flux and then resuspended in an excess of butylamine (Fluka, 99.5\%). ${ }^{7}$ Subsequently, the $\mathrm{nc}-\mathrm{PbS}$ were dissolved in toluene at a concentration of $50 \mathrm{mg} / \mathrm{ml}$ for device fabrication. Devices were fabricated on indium tin oxide (ITO) coated $(100 \mathrm{~nm})$ glass substrates; ultrasonically cleaned using a three stage process in toluene, an aqueous surfactant solution and acetone. Discrete heterojunction photovoltaic devices were fabricated in a stratified method, starting with the sublimation of pentacene under vacuum $\left(1 \times 10^{-5}\right.$ Torr $)$ at a deposition rate of $\sim 0.1 \mathrm{~nm} \mathrm{~s}^{-1}$ on to precleaned ITO coated glass substrates. Subsequently, the deposited pentacene layer was nanoimprinted using a NIL system (Obducat AB, Sweden) at $4 \mathrm{MPa}$ applied pressure utilizing a laser textured silicon stamp. A $50 \mathrm{mg} / \mathrm{ml}$ nc- $\mathrm{PbS}$ solution in toluene was spin cast onto the nanoimprinted pentacene layer at $700 \mathrm{rpm}$. Afterward, devices with an active device area of $8-10 \mathrm{~mm}^{2}$ were fabricated by evaporating an $\mathrm{Al}$ top contact. Reference pentacene/nc$\mathrm{PbS}$ photovoltaic devices were fabricated without nanoimprinting.

A KrF Lambda Physik excimer laser (LPX 210i), operating at $248 \mathrm{~nm}$ with $25 \mathrm{~ns}$ full width half maximum pulse duration, was used for nanostructuring $\sim 400 \mathrm{~nm}$ thick amorphous silicon film on glass which was used as the imprinting stamp for this work. A $4 \times 10 \mathrm{~mm}^{2}$ excimer laser pulse with an asymmetrical peak profile along the length and a constant energy along the width was scanned along the long direction over the sample. The steeper edge of the pulse was used deliberately as the leading edge to increase the surface roughness. ${ }^{8}$ The samples were irradiated in air, mounted on a translation stage for large area irradiation. A $0.2 \mathrm{~mm}$ overlap was maintained between the two scan lines, resulting in a uniformly nanostructured $2.5 \times 2.0 \mathrm{~cm}^{2}$ nanocrystalline $\mathrm{Si}$ stamp. The $222 \mathrm{~mJ} \mathrm{~cm}^{-2}$ laser pulse was scanned at $2.5 \mathrm{~mm} / \mathrm{s}$ with varying pulse repetition rate. Current-voltage characterization of the photovoltaic cells was conducted under AM1.5 simulated solar irradiation (Oriel 81160 Class B simulator) using a Keithley 2400 sourcemeter. A Digital instrument Dimension 3100 scanning probe microscope in atomic force microscopy (AFM) and conductive (C-) AFM modes was used to investigate the surface morphology and conductivity changes of the pentacene films. An FEI Quanta 


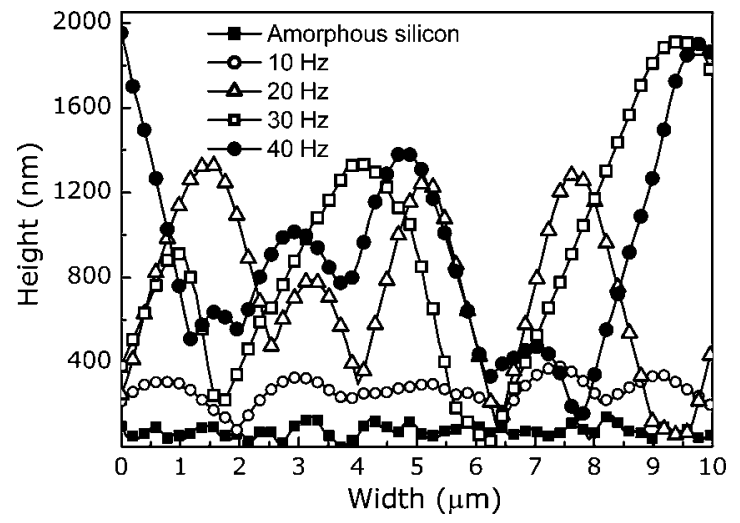

FIG. 1. Height profiles of initial Si substrate and subsequently laser textured at $222 \mathrm{~mJ} / \mathrm{cm}^{2}$ with different pulse repetition rates. Amorphous Si (black squares), $10 \mathrm{~Hz}$ (hollow circles), $20 \mathrm{~Hz}$ (hollow triangles), $30 \mathrm{~Hz}$ (hollow squares) and $40 \mathrm{~Hz}$ (black circles).

200F scanning electron microscope (SEM) was used to investigate and estimate the feature size of the textured $\mathrm{Si}$ stamp.

Figure 1 shows the height profiles of Si stamps fabricated using excimer laser at different pulse repetition rates. A concomitant increase of the average height and rms roughness with the pulse repetition rate is observed. The stamp fabricated at $40 \mathrm{~Hz}$ which was used for imprinting pentacene, resulted in $1114 \mathrm{~nm}$ average height and $559 \mathrm{~nm} \mathrm{rms}$ roughness. The scanning electron micrograph shown in Fig. 2 shows the uniformity of the features obtained from laser texturing.

Current density-voltage $(J-V)$ characteristics of the imprinted and reference hybrid devices are shown in Fig. 3. Imprinted pentacene/nc-PbS device demonstrates a $J_{\mathrm{sc}}$ of $0.27 \mathrm{~mA} / \mathrm{cm}^{2}$, which is two orders of magnitude greater than the reference pentacene/nc-PbS device (Fig. 3, inset). The $V_{\mathrm{oc}}$ is also observed to be noticeably increased by $0.17 \mathrm{~V}$ after nanoimprinting. Interestingly, $J_{\mathrm{sc}}$ of an imprinted pentacene-only device (ITO/pentacene/Al) $\left(0.008 \mathrm{~mA} / \mathrm{cm}^{2}\right)$ was smaller than the $J_{\mathrm{sc}}$ of a reference nonimprinted

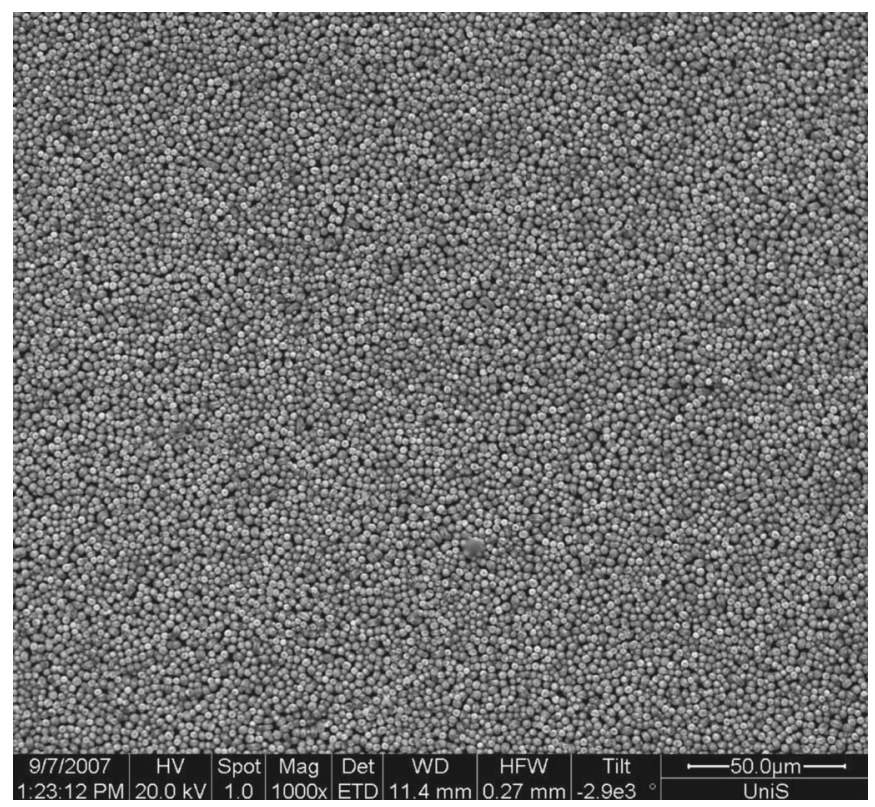

FIG. 2. Scanning electron micrograph $\left(275 \times 240 \mu \mathrm{m}^{2}\right)$ of a laser textured

stamp at $40 \mathrm{~Hz}$, with a $222 \mathrm{~mJ} / \mathrm{cm}^{2}$ energy density.
Downloaded 30 Mar 2009 to 131.227 .178 .132 . Redistribution subject to AIP license or copyright; see http://

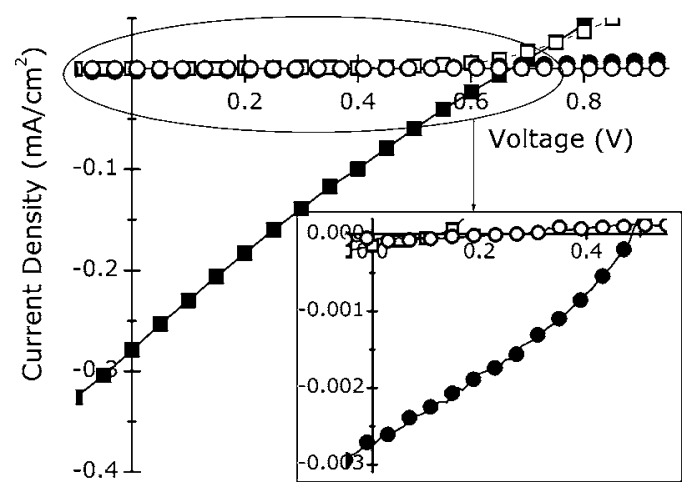

FIG. 3. Current density-voltage characteristics of imprinted (black squares: light; hollow squares: dark) and reference (black circles: light; hollow circles: dark) devices.

pentacene-only device $\left(0.013 \mathrm{~mA} / \mathrm{cm}^{2}\right)$, as shown in Fig. 4(a). The reduction after imprinting can be due to several reasons, including vacuum break during processing for the imprinting stage. However, the pentacene/nc-PbS device shows marked improvement in performance compared to pentacene-only devices, suggesting that the effect of nanoimprinting of pentacene is beneficial only when used in a heterojunction system. Therefore, to understand the mechanism of $J_{\mathrm{sc}}$ improvement by nanoimprinting of the pentacene/nc$\mathrm{PbS}$ hybrid device, comparison is carried out to an alternative heterojunction pentacene/ $\mathrm{C}_{60}$ system. ${ }^{5}$ The improvement of $J_{\mathrm{sc}}$ of pentacene/nc-PbS cell (two orders of magnitude) upon nanoimprinting is significant compared to

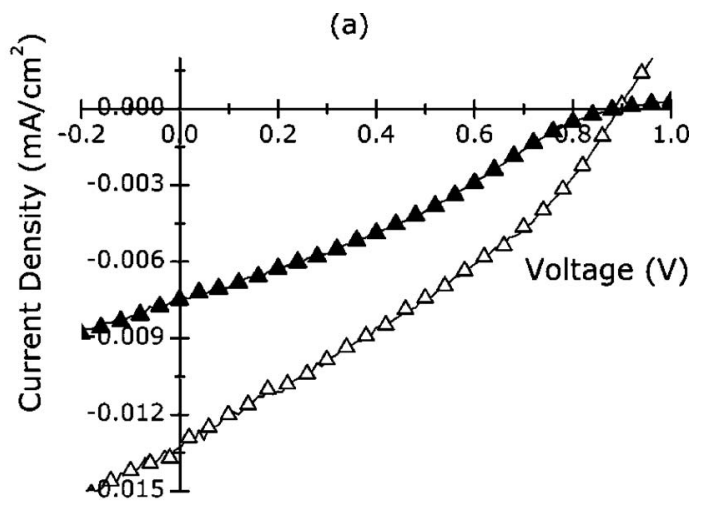

(b)

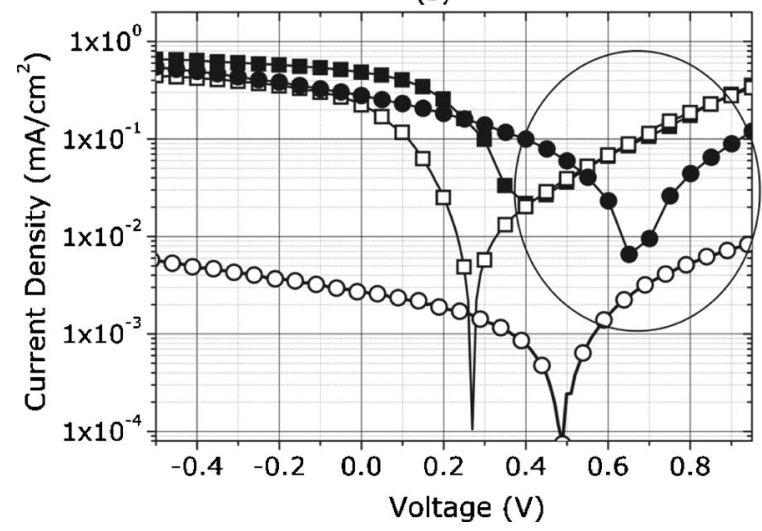

FIG. 4. (a) Current density-voltage characteristics of ITO/pentacene/Al devices (black triangles: imprinted; hollow triangles: reference) (b) Current density-voltage characteristics of pentacene $/ \mathrm{C}_{60}$ (Ref. 5) (black squares: imprinted; hollow squares: reference) and pentacene/nc-PbS (black circles: imprinted; hollow circles: reference) devices

AIP license or copyright; see http://apl.aip.org/apl/copyright.jsp 
nanoimprinted pentacene $/ \mathrm{C}_{60}$ devices with a similar process (twofold increase in $J_{\text {sc }}$ ). ${ }^{5}$ Figure 4(b) compares the $J$ - $V$ characteristics of nanoimprinted pentacene/nc-PbS and pentacene $/ \mathrm{C}_{60}$ devices, along with the respective reference devices under simulated sunlight. From Fig. 4(b), it can be seen that under forward bias [area shown by a circle in Fig. 4(b)], both imprinted and reference pentacene $/ \mathrm{C}_{60}$ devices show nearly identical $J$ - $V$ characteristics (coincidental dark and white square plots). This observation is contrary to pentacene/nc-PbS devices which are clearly evident from Fig. 4(b). The imprinted pentacene/nc-PbS device demonstrates higher current densities in forward bias, by an order of magnitude compared to the reference device (Fig. 4(b): dark and white circle plots).

The postnanoimprinting increase of the short circuit current in the pentacene $/ \mathrm{C}_{60}$ devices were attributed to the increased interfacial area facilitating enhanced charge dissociation. It was observed that when imprinted using a stamp having an average height (rms roughness) of 23(9) nm, the pentacene film morphology converted from a relatively smooth film at $27(6) \mathrm{nm}$ to a textured one at $48(16) \mathrm{nm}$, indicating increase of the active surface area. ${ }^{5}$ However, the laser textured $\mathrm{Si}$ stamp used for pentacene/nc-PbS device fabrication demonstrates a significantly greater average height of features (rms roughness) of 1114(559) nm. In order to quantify surface modifications after imprinting, AFM was performed on $75 \mathrm{~nm}$ thick pentacene films deposited on ITO coated glass and imprinted with an identical imprinting process used for pentacene/nc-PbS device fabrication. The average height and rms roughness of the as-deposited pentacene film were measured as 18.6 and $4.3 \mathrm{~nm}$, respectively. The imprinted pentacene results in an average height of $18.3 \mathrm{~nm}$ and an rms roughness of $3.9 \mathrm{~nm}$, which are extremely close to the as-deposited film's values. Therefore, interfacial area improvement in pentacene/nc-PbS after nanoimprinting can be regarded as insignificant. Hence, an alternative phenomenon must occur for the observed $J_{\mathrm{sc}}$ improvement of pentacene/nc-PbS devices upon nanoimprinting.

It has been reported that changes in interatomic spacing through applied high pressures result in changes in electronic properties of acenes, both theoretically and experimentally. ${ }^{9-11}$ The ncrease of mobility has been reported for pentacene at hydrostatic pressures in gigapascal range. ${ }^{9}$ In order to investigate possible changes in carrier mobility, C-AFM was performed on $75 \mathrm{~nm}$ as-deposited and imprinted pentacene films as per AFM analysis. The average and rms currents of the as-deposited pentacene films were measured as 29.9 and $19.4 \mathrm{pA}$, respectively. For imprinted pentacene, the average and rms currents show a fivefold increase at 163.9 and $96.8 \mathrm{pA}$, respectively. This result is clear evidence that conductivity of pentacene films improve upon nanoimprinting with large aspect ratio stamps. Hence, a hypothesis can be put forward to explain the increase in the photocurrent in the nanoimprinted hybrid devices by proposing that the dramatic increase of the $J_{\mathrm{sc}}$ of imprinted pentacene/nc-PbS, as compared to a reference, is caused by the increase of the charge mobility within the pentacene layer by nanoimprinting.

A careful analysis of the stamp used for the work using SEM and AFM allows calculating the effective localized pressure applied on pentacene films during imprinting. AFM analysis of the stamp shows that the average feature height is $1114 \mathrm{~nm}$. From the electron micrographs, the average base diameter of the features can be calculated as $2170 \mathrm{~nm}$. Assuming a conical shape for the features, and counting the number of features in a known area, the planer area of the stamp at $75 \mathrm{~nm}$ (thickness of pentacene used) from the top was calculated to be $1.4 \%$ of total area of the stamp. Since the pressure applied on the stamp during the imprinting process is $4 \mathrm{MPa}$, the effective pressure at each tip calculates to be $0.286 \mathrm{GPa}$ as a minimum value. This result is consistent with the work reported by Rang et al., who demonstrated hydrostatic pressure induced increase in photoconductivity in single crystal pentacene films up to $0.3 \mathrm{GPa}$, caused by the increasing overlap of the adjacent molecular orbitals caused by pressure induced decrease of the intermolecular distances. ${ }^{9}$ Increased mobility allows for thicker acene layers in organic photovoltaics, which would aid improved efficiency due to enhanced light absorption. Furthermore, the potential for other electronic applications is significant such as organic thin film transistors. Thin film transistors with very thin organic layers maybe possible with significantly improved performances.

In conclusion, nanoimprinted pentacene/nc-PbS hybrid solar cells were fabricated using excimer laser textured silicon stamp resulting in two orders of magnitude higher short circuit current densities. The improvement of the device performance is attributed to the improved electronic transport properties of the imprinted pentacene layer due to localized high pressures leading to decrease intermolecular distances of the small molecule organic film.

This work was supported by the Portfolio Partnership Award from EPSRC, U.K. The authors acknowledge Dr. Thierry Lutz for providing PbS quantum dots for this work.

${ }^{1}$ J. Y. Kim, K. Lee, N. E. Coates, D. Moses, T. Nguyen, M. Dante, and A. J. Heeger, Science 317, 222 (2007)

${ }^{2}$ D. M. N. M. Dissanayake, R. A. Hatton, T. Lutz, C. E. Giusca, R. J. Curry, and S. R. P. Silva, Appl. Phys. Lett. 91, 133506 (2007).

${ }^{3}$ D. M. N. M. Dissanayake, R. A. Hatton, T. Lutz, C. E. Giusca, R. J. Curry, and S. R. P. Silva (unpublished).

${ }^{4}$ E. J. D. Klem, D. D. MacNeil, P. W. Cyr, L. Levina, and E. H. Sargent, Appl. Phys. Lett. 90, 183113 (2007).

${ }^{5}$ D. M. N. M. Dissanayake, A. A. D. T. Adikaari, R. J. Curry, R. A. Hatton, and S. R. P. Silva, Appl. Phys. Lett. 90, 253502 (2007).

${ }^{6}$ M. A. Hines and G. D. Scholes, Adv. Mater. (Weinheim, Ger.) 15, 1844 (2003).

${ }^{7}$ G. Konstantatos, I. Howard, A. Fischer, S. Hoogland, J. Clifford, E. Klem, L. Levina, and E. H. Sargent, Nature (London) 442, 180 (2006).

${ }^{8}$ A. A. D. T. Adikaari, N. K. Mudugamuwa, and S. R. P. Silva, Appl. Phys. Lett. 90, 171912 (2007).

${ }^{9}$ Z. Rang, A. Haraldsson, D. M. Kim, P. P. Ruden, R. J. Chesterfied, and C. D. Frisbie, Appl. Phys. Lett. 79, 2731 (2001).

${ }^{10}$ M. Chandrasekhar, S. Guha, and W. Graipner, Adv. Mater. (Weinheim, Ger.) 13, 613 (2001).

${ }^{11}$ M. Oehzelt, A. Aichholzer, R. Resel, G. Heimel, E. Venuti, and R. G. Della Valle, Phys. Rev. B 74, 104103 (2006). 